\title{
C-SELFADJOINTNESS OF THE PRODUCT OF A COMPOSITION OPERATOR AND A MAXIMAL DIFFERENTIATION OPERATOR
}

\author{
Mahmood Haji ShaAbani, Mahsa Fatehi* And Pham Viet Hai
}

Abstract. Let $\varphi$ be an automorphism of $\mathbb{D}$. In this paper, we consider the operator $C_{\varphi} D_{\psi_{0}, \psi_{1}}$ on the Hardy space $H^{2}$ which is the products of composition and the maximal differential operator. We characterize these operators which are $C$-selfadjoint with respect to some conjugations $C$. Moreover, we find all hermitian operators $C_{\varphi} D_{\psi_{0}, \psi_{1}}$, when $\varphi$ is a rotation.

Mathematics subject classification (2010): 47B33, 47E99.

Keywords and phrases: Hardy space, differential operator, composition operator, conjugation, complex symmetric operator, hermitian.

\section{REFERENCES}

[1] P. S. Bourdon And S. W. Noor, Complex symmetric of invertible composition operators, J. Math. Anal. Appl. 429 (2015), 105-110.

[2] C. C. CowEn, Linear fractional composition operators on $H^{2}$, Integral Equations and Operator Theory 11 (1988), 151-160.

[3] C. C. Cowen And B. D. MacCluer, Composition Operators on Spaces of Analytic Functions, Studies in Advanced Mathematics, CRC Press, Boca Raton, FL, 1995.

[4] M. FATEHI, Complex symmetric weighted composition operators, Complex Var. Elliptic Equ. 64 (2019), 710-720.

[5] M. Fatehi and C. Hammond, Composition-Differentiation operators on the Hardy space, Proc. Amer. Math. Soc. 148 (2020), 2893-2900.

[6] S. R. GARCIA AND C. HAMMOND, Which weighted composition operators are complex symmetric?, Oper. Theory Adv. Appl. 236 (2014), 171-179.

[7] S. R. Garcia And M. Putinar, Complex symmetric operators and applications, Trans. Amer. Math. Soc. 358 (2006), 1285-1315.

[8] S. R. Garcia And M. Putinar, Complex symmetric operators and applications II, Trans. Amer. Math. Soc. 359 (2007), 3913-3931.

[9] S. R. Garcia, E. Prodan AND M. Putinar, Mathematical and physical aspects of complex symmetric operators, J. Phys. A: Math. Theor. 47 (2014): 353001.

[10] P. V. HAI, Complex symmetry of first-order differential operators on the Hardy space, arXiv:1901.02331v1.

[11] P. V. HAI AND M. PUtinaR, Complex symmetric differential operators on Fock space, J. Differential Equations, 265 (2018), 4213-4250.

[12] S. Jung, Y. Kim, E. Ko, AND J. E. LeE, Complex symmetric weighted composition operators on $H^{2}(\mathbb{D})$, J. Funct. Anal. 267 (2014), 323-351.

[13] S. K. Narayan, D. Sievewright And D. Thompson, Complex symmetric composition operators on $H^{2}$, J. Math. Anal. Appl. 443 (2016), 625-630.

[14] S. OHno, Products of composition and differentiation between Hardy spaces, Bull. Austral. Math. Soc. 73 (2006), 235-243.

[15] E. Prodan, S. R. Garcia And M. Putinar, Norm estimates of complex symmetric operators applied to quantum systems, Journal of Physics A: Mathematical and General, 39 (2006), 389-400. 